\title{
Phenotypic variability in a Hungarian patient with the $4 q 21$ microdeletion syndrome
}

\author{
Katalin Komlósi ${ }^{1,3+}$, Balázs Duga ${ }^{1,3+}$, Kinga Hadzsiev ${ }^{1,3}$, Márta Czakó1,3, György Kosztolányi ${ }^{1,3}$, András Fogarasi $^{2}$ \\ and Béla Melegh ${ }^{1,3^{*}}$
}

\begin{abstract}
Background: Interstitial deletions of 4q21 (MIM 613509) have already been reported in more than a dozen patients with deletions ranging from 2 to $15.1 \mathrm{Mb}$ delineating a common phenotype including marked growth restriction, hypotonia, severe developmental delay with absent or delayed speech and distinctive facial features. A minimal critical region of $1.37 \mathrm{Mb}$ accounting for the common features with 5 known genes (PRKG2, RASGEF1B, HNRNPD, HNRPDL, and ENOPH1) has been described so far.
\end{abstract}

Results: Here we report on a 5 year-old Hungarian girl presenting with severe developmental delay, good receptive language but absent spoken speech, short stature, dystrophy, hypotonia, distinctive facies including broad forehead, frontal bossing, downward slanting palpebral fissures, hypertelorism, hypoplastic ear-lobes, anteverted nostrils, short philtrum, small mouth, higharched palate, short, small hands and feet, distally narrowing fingers and clinodactyly. Cerebral MRI showed ventricular dilation and an increase in periventricular signal intensity. After extensive metabolic tests and exclusion of subtelomeric deletions array CGH analysis was performed using the Agilent Human Genome G3 SurePrint 8x60K Microarray (Agilent Technologies, USA), which detected a 4,85 Mb de novo interstitial deletion of $4 q 21.21-4 q 21.23$. The clinical symptoms only partly overlap with reported $4 q 21$ microdeletion cases. Among multiple annotated genes our patient is also haploinsufficient for the following genes: RASGEF1B being a strong candidate for the neurodevelopmental features and PRKG2 for severe growth delay.

Conclusion: The first Hungarian case of 4q21 deletion adds to the phenotypic spectrum of this novel microdeletion syndrome and underlines the importance of array CGH to uncover the heterogeneous causes of intellectual disability.

Keywords: Submicroscopic deletion, Array CGH, 4q21, Short stature, Intellectual disability

\section{Background}

The recent wide-spread use of microarray-based comparative genomic hybridization (array CGH) has extensively aided the elucidation of the underlying cause in patients with severe developmental delay and intellectual disability with dysmorphic features [1]. Interstitial deletions of 4q21 have been reported in about a dozen patients [1-8] with deletions ranging from 2 to $15.1 \mathrm{Mb}$ delineating a common phenotype including marked growth restriction, hypotonia, severe developmental delay with absent or

\footnotetext{
* Correspondence: melegh.bela@pte.hu

${ }^{\dagger}$ Equal contributors

'Department of Medical Genetics, Clinical Centre, University of Pecs, Szigeti

Street 12, Pecs H-7624, Hungary

${ }^{3}$ Szentágothai Research Centre, University of Pecs, Ifjusag Street 20, Pecs

H-7624, Hungary

Full list of author information is available at the end of the article
}

delayed speech, small hands and feet and distinctive facial features as broad forehead, hypertelorism, and prominent central incisors. A minimal critical region of $1.37 \mathrm{Mb}$ accounting for the common features with 5 known genes (PRKG2, RASGEF1B, HNRNPD, HNRPDL, ENOPH1) has been described so far [5].

Here, we report the first Hungarian case of 4q21 deletion adding to the phenotypic spectrum of this novel microdeletion syndrome.

\section{Case presentation \\ Results}

After extensive metabolic tests and exclusion of subtelomeric deletions array CGH analysis was performed using the Agilent Human Genome G3 SurePrint 8x60K Microarray, which detected a $4,85 \mathrm{Mb}$ de novo interstitial deletion of 4q21.21-4q21.23 (ch4:81 408 980-86 261 953) 
(Figure 1). The deletion in our patient involved the following genes: PRKG2 (MIM 601591), RASGEF1B (MIM 614532), HNRNPD (MIM 607137), HNRPDL, ENOPH1, COQ2, MRPS18C, THAP9, HPSE, and CDS1. Except for known CNVs, no copy number alterations were observed in other chromosomes (data not shown). Based on the normal CGH array profile of the parents this deletion proved to be de novo. The deletion was not reported as polymorphic or structural variant in the publicly available databases.

\section{Discussion}

The widespread use of array CGH has led to the delineation of many novel entities associated with developmental delay [1]. Beyond revealing the underlying cause it provides information for prognosis, the medical management of the symptoms and access to resources for the affected families, and also gives the basis for estimating recurrence risks.

Several reports and studies have delineated a 4q21 microdeletion syndrome with a common phenotype including marked growth restriction, hypotonia, severe developmental delay with absent or delayed speech, small hands and feet and distinctive facial features as broad forehead, hypertelorism, and prominent lower and upper incisors [5]. A minimal critical region of $1.37 \mathrm{Mb}$ with 5 known genes (PRKG2, RASGEF1B, HNRNPD, HNRPDL, ENOPH1) has been described so far [5]. Comparison of our case with previously published cases (Table 1) revealed several common features but also some variation in the phenotype. The $4.85 \mathrm{Mb}$ deletion in our patient includes the minimal critical region of the $4 \mathrm{q} 21$ microdeletion syndrome, encompassing the candidate genes, PRKG2 and RASGEF1B, previously described as major

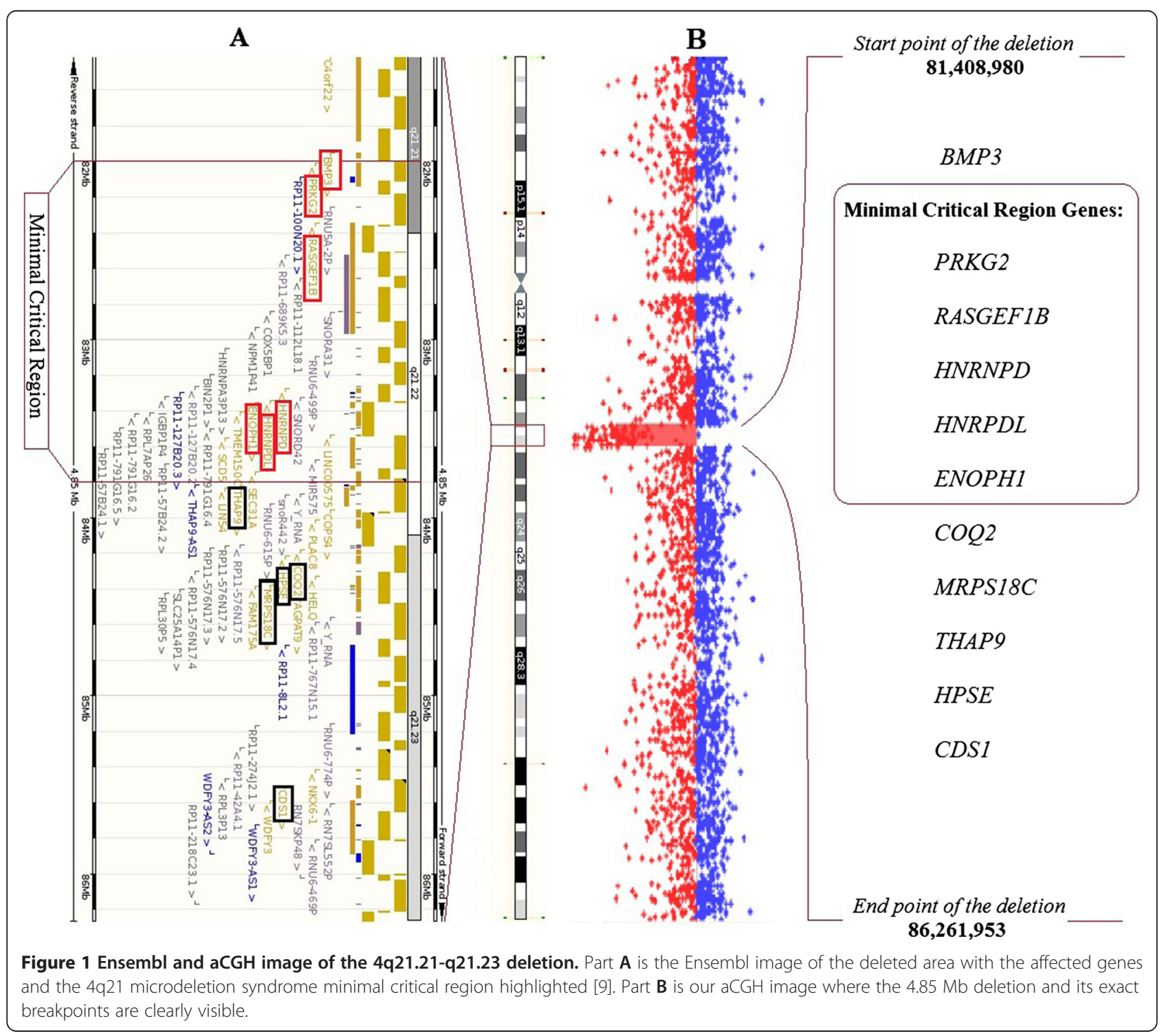




\begin{tabular}{|c|c|c|c|c|}
\hline Clinical/common features & $\begin{array}{l}\text { Minimal critical } \\
\text { region [5] }\end{array}$ & $\begin{array}{l}\text { Smallest described } \\
\text { deletion [7] }\end{array}$ & $\begin{array}{l}\text { Largest described } \\
\text { deletion [5] }\end{array}$ & Current case report \\
\hline Deletion & $4 q 21.21-21.22$ & $4 q 21.22-q 21.23$ & $4 q 21.21-q 22.3$ & $4 q 21.21-4 q 21.23$ \\
\hline Size (Mb) & 1.37 & 2.0 & 15.1 & 4.85 \\
\hline Age at diagnosis (years) & NA & 7 & 8 & 5 \\
\hline \multicolumn{5}{|l|}{ Craniofacial features } \\
\hline Frontal bossing, broad forehead & Yes & Yes & Yes & Yes \\
\hline Downslanting palpebral fissures & ND & Yes & ND & Yes \\
\hline Hypertelorism & Yes & Yes & No & Yes \\
\hline Anteverted nostrils & ND & ND & Yes & Yes \\
\hline Short philtrum & ND & ND & Yes & Yes \\
\hline Hypoplastic ear-lobes & ND & No & Yes & Yes \\
\hline Small mouth & ND & Yes & ND & Yes \\
\hline Higharched palate & ND & Yes & ND & Yes \\
\hline Developmental delay & Yes & Moderate & Yes & Yes \\
\hline Neonatal hypotonia & Yes & No & Yes & Yes \\
\hline Gross motor delay & Yes & moderate & Yes & Yes \\
\hline Delayed speech & Yes & Yes & Yes & Yes \\
\hline Stereotypic movements & ND & ND & ND & Yes \\
\hline Behavioral disturbance & ND & Yes & ND & Yes \\
\hline Anthropometric and skeletal abnormalities & Yes & Yes & Yes & Yes \\
\hline IUGR & ND & No & Yes & Yes \\
\hline Birth weight (centile) & ND & 50th & 25th & 25-50th \\
\hline Postnatal growth delay & Yes & No & $-5 S D$ & $-2 \mathrm{SD}$ \\
\hline Conserved head circumference & Yes & $+1 S D$ & $-0.5 \mathrm{SD}$ & $+1 S D$ \\
\hline Small hands and small feet & Yes & Yes & No & Yes \\
\hline Brachydactyly & Yes & Yes & No & Yes \\
\hline Cerebral imaging abnormality & & & Yes & Yes \\
\hline Ventricular dilation & ND & No & Yes & Yes \\
\hline Corpus callosum hypoplasia & ND & No & ND & Yes \\
\hline Cerebellar vermis hypoplasia & ND & No & ND & No \\
\hline Frontal cerebral hypoplasia & ND & No & Yes & No \\
\hline
\end{tabular}

Yes: feature present; no: feature absent; ND: data not accessible, NA: not applicable.

determinants of the 4q21 phenotype. Our patient shares haploinsufficiency with the patient portrayed as having the smallest reported deletion in this region, a novel 2.0 $\mathrm{Mb}$ deletion encompassing three of the genes in the proposed minimal critical region: HNRNPD, HNRPDL, and ENOPH1 [7]. The shared features between this patient and our patient, including macrocephaly, small hands and feet, developmental delay, and the distinctive facial features of broad forehead, hypertelorism and prominent lower incisors, stress the role of these genes as likely candidates for the shared phenotype through yet unrevealed mechanisms [7].
Among the dysmorphic features the characteristic brachydactyly observed in other patients was less pronounced in our index patient, and no shorter 2nd toe was observed as described in the classic phenotype. Joint laxity and hypermobile joints, also observed in our patient, have been described recently in a patient with a proximal 4q interstitial deletion of $24.89 \mathrm{Mb}$ encompassing 4q12-4q21.21 [10] and is only known in 3 additional patients with proximal $4 \mathrm{q}$ deletions. In addition to the common neurocognitive characteristics seen in most 4q21 cases our patient also exhibited stereotypic movements and a behavioral disturbance including occasional 
self-injurious behavior and aggression towards others as described in the patient with the $2 \mathrm{Mb}$ deletion [7]. It is also noteworthy that good receptive language and communication by sign was observed in our patient besides absent speech also resembling the described case with a large proximal 4q deletion [10], however, more studies with the precise description of the deletion boundaries will be needed to point out genes responsible for the overlapping features.

The 4,85 Mb region involved in the deletion contains a number of genes, some of which have already been discussed as being major determinants of the phenotype $[1,5]$, while the role of other genes and their impact on the phenotype still need to be elucidated. We have learned from previous works that haploinsufficiency of the minimal critical region is essential for the expression of the classic 4q21 phenotype, within this region, the genes PRKG2 and RASGEF1B, have been identified as major determinants in the development of the characteristic features [5]. RASGEF1B encodes a highly conserved guanine nucleotide exchange factor for Ras family proteins. This protein superfamily is involved in various basic cellular functions such as signal transduction, cytoskeleton dynamics and intracellular trafficking. It is highly expressed in the central nervous system, and may play a role in actin and microtubule dynamics regulating both dendrite and spine structural plasticity [11]. Since several genes related to intellectual disability have been identified in the Rho-GTPase signaling pathway, RAS$G E F 1 B$ seems to play a role in the cognitive features of the $4 \mathrm{q} 21$ phenotype [5].

There is strong evidence that the second basic feature of the microdeletion syndrome, severe growth delay, can be attributed to the PRKG2 gene which encodes a cGMP-dependent protein kinase type II protein. Mice with a null mutation of this gene developed postnatal dwarfism as a result of severe endochondral ossification defect at the growth plates and impaired chondrocyte growth. While small hands, short fingers and feet were described, no postnatal growth delay was observed in the patient with the smallest described deletion [7] not containing the PRKG2 gene, while in patients deleted for the minimal critical region and in our current case growth delay was severe (Table 1). Those observations also underline that the haploinsufficiency of the PRKG2 gene could explain growth failure [7] in most of the 4q21 patients. On the other hand, haploinsufficiency of PRKG2 has previously also been linked to severe cognitive developmental delay [12].

Additionally the deletion in our patient also involved several genes (BMP3, COQ2, MRPS18C, THAP9, HPSE, and $C D S 1$ ) for which no direct function can be linked to the features observed in our patient. In rat embryos, Bmp3 was suggested to be involved in pattern formation during early skeletal development [13]. In Bmp3 -/embryos or newborns no skeletal defects were found, only increased trabecular metaphyseal bone density and total trabecular bone volume [14]. On the other hand, a missense mutation in the BMP3 gene (F452L) was associated with cranioskeletal differences in canines [15]. Strehle et al. argued that haploinsufficiency of $B M P 3$ might be associated with short stature and other skeletal anomalies in 4q21 interstitial deletions [16]. Thus, it cannot be ruled out that haploinsufficiency of $B M P 3$ may also contribute to the cranial features observed in our patient, such as broad forehead and frontal bossing.

The HPSE gene encodes a heparanase belonging to the family of endoglycosidases which cleave the heparan sulfate side chain of heparan sulfate proteoglycans (HSPGs) and contribute to the remodeling of the extracellular matrix for cell movement or the release of bioactive molecules from the extracellular matrix or cell surface [17]. Vlodavsky et al. demonstrated a correlation of HPSE expression and heparanase activity with increased metastatic potential in breast cancer tissues and cell lines [18]. Currently no direct function of the HPSE gene can be linked to developmental disorders, however, it can be assumed that the basic function of extracellular matrix remodeling might also be essential for neurodevelopment. Further detailed case reports or experimental data are needed to learn more about the clinical relevance of those genes.

\section{Conclusions}

We describe the first Hungarian patient with a de novo previously unreported interstitial $4 \mathrm{q}$ deletion, syndromic severe developmental delay, absence of spoken language and behavioral disturbance. The clinical symptoms in our patient partially overlap with reported 4q21 microdeletion cases. Among the multiple annotated genes our patient is also haploinsufficient for $R A S G E F 1 B$, a strong candidate for the neurodevelopmental features and PRKG2 for severe growth delay. In the future elucidation of the clinical relevance of several other deleted genes in 4q21 patients may help establish guidelines for adequate healthcare management of those patients. Our case of $4 \mathrm{q} 21$ deletion adds to the phenotypic spectrum of this novel microdeletion syndrome and underlines the importance of array CGH to uncover the heterogeneous causes of intellectual disability.

\section{Methods}

\section{Patient report}

The patient was a 5 year old girl born by caesarean section at 39th week of gestation as the second child of non-consanguineous healthy Hungarian parents, the family history was unremarkable. Her birth weight was $2750 \mathrm{~g}(25-50 \mathrm{pc})$, her length $49 \mathrm{~cm}(5-10 \mathrm{pc})$, the head 
circumference $36 \mathrm{~cm}(+1 \mathrm{SD})$. Her 5 and 10 minute Apgar scores were 9/10. In the perinatal period mild icterus, joint laxity in the hips, axial hypotonia and poor feeding was noted. At 1 week of age severe axial hypotonia and spasticity in the lower limbs was recognized and there was only slight improvement following extensive neurohabilitation. After 3 months her somatic and psychomotor development slowed down and has been very slow ever since. At 6 months of age the patient was hospitalized with severe obstructive bronchitis and during her first year she suffered several upper airway infections with dense mucous and chronic diarrhea, but CFTR-related diseases were excluded. At 14 months of age brain MRI revealed significantly widened and abnormally structured ventricles, diminished periventricular white matter and hypoplasia of the corpus callosum. At the age of 18 month the patient was referred to our genetic counseling unit because of severe hypotonia and developmental delay. Postnatal growth delay: weight was $9.5 \mathrm{~kg}(5-10 \mathrm{pc})$, height $68 \mathrm{~cm} \mathrm{(<3} \mathrm{pc)} \mathrm{and} \mathrm{head} \mathrm{circum-}$ ference $48.5 \mathrm{~cm}(+1 \mathrm{SD})$ and a distinctive facies including broad forehead, frontal bossing, downward slanting palpebral fissures, hypertelorism, hypoplastic ear-lobes, anteverted nostrils, short philtrum, small mouth, higharched palate as well as short, small hands and feet, distally narrowing fingers, clinodactyly and joint laxity were noted. Neurological examination revealed severe generalized hypotonia and absent speech development. Gross motor milestones were severely delayed despite of extensive neurohabilitation: at the age of 2.5 years she was unable to sit alone, she did not crawl and was unable to stand alone. At the age of 5 years she was able to walk, sit alone, but had no speech. She had good receptive language and used signs and gestures to communicate but had no speech. Stereotypical movements such as hand clapping and flapping and a behavioral disturbance, including occasional self-injurious behavior and aggression toward others were observed. Epilepsy has not been noted so far and repeated EEGs gave negative results. Extensive metabolic (carnitine-ester profiling, amino acids, urine organic acids, isoelectric focusing for CDGs) and genetic testing (routine karyotyping, CFTR sequencing, mitochondrial mutation screening) yielded negative results.

\section{Array Comparative Genomic Hybridization (aCGH) analysis}

Array CGH was performed using the Agilent Human Genome G3 SurePrint 8x60K Microarray (Agilent Technologies, USA), a high resolution 60-mer oligonucleotide based microarray containing 55.077 60-mer probes, spanning coding and non-coding genomic sequences with median spacing of $33 \mathrm{~kb}$ and $41 \mathrm{~kb}$, respectively.
Purification of the DNA from blood was performed using the DNA Purification Kit NucleoSpin ${ }^{\circ}$ Dx Blood (Macherey-Nagel, Germany) according to the manufacturer's protocol. Concentration and purity of the extracted DNA were measured with the NanoDrop spectrophotometer (NanoDrop Technologies, Inc.). Pooled genomic DNA from peripheral blood leukocytes of phenotypically normal males or females from Promega was used as a reference (Promega Male/Female Reference DNA, Promega Corporation, USA).

Labeling and hybridization were carried out based on the Agilent protocol (Agilent Oligonucleotide Array-Based CGH for Genomic DNA Analysis - Enzymatic Labeling Protocol v7.2; July 2012). Array image was acquired using an Agilent laser scanner G2565CA (Agilent Technologies, California, USA) and analyzed with the Agilent Feature Extraction software (v10.10.1.1.). Results were presented by Agilent Cytogenomics software (v2.5.8.11). DNA sequence information refers to the public UCSC database (Human Genome Browser, Feb 2009 Assembly; GRCh37: hg19).

The deletion detected was aligned to known aberrations listed in publicly available databases, such as the DECIPHER (Database of Chromosomal Imbalance and Phenotype in Humans using Ensembl Resources) [19], DGV (the Database of Genomic Variants) [20], Ensembl [21] and ECARUCA [22]. Parental samples were analyzed using the same array and method.

\section{Consent}

Written informed consent was obtained from the patient for publication of this Case report and any accompanying images. A copy of the written consent is available for review by the Editor-in-Chief of this journal.

\section{Abbreviations}

MRI: Magnetic Resonance Image; a(rray) CGH: Array Comparative Genomic Hybridization; EEG: Electroencephalography; CDG: Congenital Disorder of Glycosylation; CFTR: Cystic fibrosis transmembrane conductance regulator; GRCh37:hg19: Genome Reference Consortium human 37/human genome 19; UCSC database: University of California, Santa Cruz Genome Browser database; DECIPHER: Database of Chromosomal Imbalance and Phenotype in Humans using Ensembl Resources; DGV: The Database of Genomic Variants; ECARUCA: European Cytogeneticists Association Register of Unbalanced Chromosome Aberrations; CNV: Copy Number Variation.

\section{Competing interests}

The authors declare that they have no competing interests.

\section{Authors' contributions}

$\mathrm{KK}, \mathrm{KH}$ and AF were responsible for the patient's clinical genetic examination. $\mathrm{KK}$ and $\mathrm{KH}$ contributed to the clinical description. BM, MCz and BD conceived and designed the molecular experiments. $M C z$ and $B D$ performed the array $\mathrm{CGH}$ and analyzed the data. $\mathrm{KK}, \mathrm{KH}, \mathrm{MCz}, \mathrm{BD}$, GyK and BM co-wrote the manuscript. BM revised the manuscript critically for important intellectual content. All authors read and approved the final manuscript.

Authors' information

Katalin Komlosi and Balazs Duga both should be considered $1^{\text {st }}$ authors. 


\section{Acknowledgements}

This work was supported by the OTKA 103983 grant to BM, the European Union and the State of Hungary, co-financed by the European Social Fund in the framework of TÁMOP-4.2.4.A/2-11/1-2012-0001 'National Excellence Program'to BD and supported by the János Bolyai Research Scholarship of the Hungarian Academy of Sciences to KK. We are grateful to the family for their cooperation.

\section{Author details}

${ }^{1}$ Department of Medical Genetics, Clinical Centre, University of Pecs, Szigeti

Street 12, Pecs H-7624, Hungary. ${ }^{2}$ Department of Neurology, Bethesda

Children's Hospital, Bethesda Street 3, Budapest H-1146, Hungary.

${ }^{3}$ Szentágothai Research Centre, University of Pecs, Ifjusag Street 20, Pecs

H-7624, Hungary.

Received: 17 November 2014 Accepted: 13 February 2015

Published online: 03 March 2015

\section{References}

1. Bartnik M, Nowakowska B, Derwinska K, Wisniowiecka-Kowalnik B, Kedzior $M$, Bernaciak J, et al. Application of array comparative genomic hybridization in 256 patients with developmental delay or intellectual disability. J Appl Genet. 2014;55(1):125-44. doi:10.1007/s13353-013-0181-x.

2. Nowaczyk MJ, Teshima IE, Siegel-Bartelt J, Clarke JT. Deletion 4q21/4q22 syndrome: two patients with de novo 4q21.3q23 and 4q13.2q23 deletions. Am J Med Genet. 1997:69(4):400-5.

3. Harada N, Nagai T, Shimokawa O, Niikawa N, Matsumoto N. A 4q21-q22 deletion in a girl with severe growth retardation. Clin Genet. 2002;61(3):226-8.

4. Friedman JM, Baross A, Delaney AD, Ally A, Arbour L, Armstrong L, et al. Oligonucleotide microarray analysis of genomic imbalance in children with mental retardation. Am J Hum Genet. 2006;79(3):500-13. doi:10.1086/ 507471

5. Bonnet C, Andrieux J, Beri-Dexheimer M, Leheup B, Boute O, Manouvrier S, et al. Microdeletion at chromosome $4 \mathrm{q} 21$ defines a new emerging syndrome with marked growth restriction, mental retardation and absent or severely delayed speech. J Med Genet. 2010;47(6):377-84. doi:10.1136/ jmg.2009.071902.

6. Dukes-Rimsky L, Guzauskas GF, Holden KR, Griggs R, Ladd S, Montoya Mdel C, et al. Microdeletion at 4q21.3 is associated with intellectual disability, dysmorphic facies, hypotonia, and short stature. Am J Med Genet. 2011;155A(9):2146-53. doi:10.1002/ajmg.a.34137.

7. Bhoj E, Halbach S, McDonald-McGinn D, Tan C, Lande R, Waggoner D, et al. Expanding the spectrum of microdeletion 4q21 syndrome: a partial phenotype with incomplete deletion of the minimal critical region and a new association with cleft palate and Pierre Robin sequence. Am J Med Genet A. 2013;161A(9):2327-33. doi:10.1002/ajmg.a.36061.

8. Zhou J, Hu P, Liu A, Li L, Ji X, Hui W, et al. [Array comparative genomic hybridization detection of a de novo 4q21.21-q22.1 deletion in a child with severe growth retardation]. Zhonghua yi xue yi chuan xue za zhi = Zhonghua yixue yichuanxue zazhi. Chinese J Med Gen. 2014;31(1):52-5. doi:10.3760/cma.j.issn. 1003-9406.2014.01.012.

9. Ensembl MapViewer. http://grch37.ensembl.org/Homo_sapiens/Location/ Overview? $\mathrm{db}=$ core\& $r=4 \% 3 \mathrm{~A} 81408980-86261953$.

10. Hemati P, du Souich C, Boerkoel CF. 4q12-4q21.21 deletion genotypephenotype correlation and the absence of piebaldism in presence of KIT haploinsufficiency. Am J Med Genet. 2015;167(1):231-7. doi:10.1002/ajmg a.36821.

11. Newey SE, Velamoor V, Govek EE, Van Aelst L. Rho GTPases, dendritic structure, and mental retardation. J Neurobiol. 2005;64(1):58-74. doi:10.1002/neu.20153.

12. Uhler MD. Cloning and expression of a novel cyclic GMP-dependent protein kinase from mouse brain. J Biol Chem. 1993:268(18):13586-91.

13. Rosen V, Wozney JM, Wang EA, Cordes P, Celeste A, McQuaid D, et al. Purification and molecular cloning of a novel group of BMPs and localization of BMP mRNA in developing bone. Connect Tissue Res. 1989;20(1-4):313-9.

14. Daluiski A, Engstrand T, Bahamonde ME, Gamer LW, Agius E, Stevenson SL, et al. Bone morphogenetic protein-3 is a negative regulator of bone density. Nat Genet. 2001;27(1):84-8. doi:10.1038/83810.
15. Schoenebeck JJ, Hutchinson SA, Byers A, Beale HC, Carrington B, Faden DL, et al. Variation of BMP3 contributes to dog breed skull diversity. PLoS Genet. 2012;8(8):e1002849. doi:10.1371/journal.pgen.1002849.

16. Strehle EM, Gruszfeld D, Schenk D, Mehta SG, Simonic I, Huang T. The spectrum of $4 q-$ syndrome illustrated by a case series. Gene. 2012;506(2):387-91. doi:10.1016/j.gene.2012.06.087.

17. McKenzie E, Tyson K, Stamps A, Smith P, Turner P, Barry R, et al. Cloning and expression profiling of $\mathrm{Hpa} 2$, a novel mammalian heparanase family member. Biochem Biophys Res Commun. 2000;276(3):1170-7. doi:10.1006/ bbrc.2000.3586.

18. Vlodavsky I, Friedmann $Y$, Elkin $M$, Aingorn $H$, Atzmon $R$, Ishai-Michaeli $R$, et al. Mammalian heparanase: gene cloning, expression and function in tumor progression and metastasis. Nat Med. 1999;5(7):793-802. doi:10.1038/10518

19. DECIPHER. [http://decipher.sanger.ac.uk]

20. the Database of Genomic Variants. [http://projects.tcag.ca/variation]

21. Ensembl. [http://wwwensembl.org]

22. ECARUCA. [http://umcecaruca01.extern.umcn.nl:8080/ecaruca/ecaruca.jsp]

\section{Submit your next manuscript to BioMed Central and take full advantage of:}

- Convenient online submission

- Thorough peer review

- No space constraints or color figure charges

- Immediate publication on acceptance

- Inclusion in PubMed, CAS, Scopus and Google Scholar

- Research which is freely available for redistribution 